Louisiana State University

LSU Digital Commons

Faculty Publications

Department of Physics \& Astronomy

$11-1-2021$

\title{
CALET Observations during the First 5 Years on the ISS
}

\author{
P. S. Marrocchesi \\ Università degli Studi di Siena \\ O. Adriani \\ Università degli Studi di Firenze \\ Y. Akaike \\ NASA Goddard Space Flight Center \\ Y. Asaoka \\ Waseda University \\ E. Berti \\ Università degli Studi di Firenze
}

See next page for additional authors

Follow this and additional works at: https://digitalcommons.Isu.edu/physics_astronomy_pubs

\section{Recommended Citation}

Marrocchesi, P., Adriani, O., Akaike, Y., Asaoka, Y., Berti, E., Bigongiari, G., Bongi, M., Brogi, P., Bruno, A., Cannady, N., Checchia, C., Cherry, M., Collazuol, G., Ficklin, A., Guzik, T., Ichimura, M., Israel, M., Kasahara, K., Kawakubo, Y., Krizmanic, J., Messineo, A., Mitchell, J., Miyake, S., Mori, M., Mori, N., Motz, H., Munakata, K., Pacini, L., Palma, F., Papini, P., Rauch, B., Ricciarini, S., \& Sakamoto, T. (2021). CALET Observations during the First 5 Years on the ISS. Physics of Atomic Nuclei, 84 (6), 985-994. https://doi.org/10.1134/ S1063778821130214

This Article is brought to you for free and open access by the Department of Physics \& Astronomy at LSU Digital Commons. It has been accepted for inclusion in Faculty Publications by an authorized administrator of LSU Digital Commons. For more information, please contact ir@lsu.edu. 


\section{Authors}

P. S. Marrocchesi, O. Adriani, Y. Akaike, Y. Asaoka, E. Berti, G. Bigongiari, M. Bongi, P. Brogi, A. Bruno, N. Cannady, C. Checchia, M. L. Cherry, G. Collazuol, A. W. Ficklin, T. G. Guzik, M. Ichimura, M. H. Israel, K. Kasahara, Y. Kawakubo, J. F. Krizmanic, A. M. Messineo, J. W. Mitchell, S. Miyake, M. Mori, N. Mori, H. M. Motz, K. Munakata, L. Pacini, F. Palma, P. Papini, B. F. Rauch, S. B. Ricciarini, and T. Sakamoto 
Louisiana State University

LSU Digital Commons

Faculty Publications

Department of Physics \& Astronomy

$11-1-2021$

\title{
CALET Observations during the First 5 Years on the ISS
}

\author{
P. S. Marrocchesi \\ Università degli Studi di Siena \\ O. Adriani \\ Università degli Studi di Firenze \\ Y. Akaike \\ NASA Goddard Space Flight Center \\ Y. Asaoka \\ Waseda University \\ E. Berti \\ Università degli Studi di Firenze
}

See next page for additional authors

Follow this and additional works at: https://digitalcommons.Isu.edu/physics_astronomy_pubs

\section{Recommended Citation}

Marrocchesi, P., Adriani, O., Akaike, Y., Asaoka, Y., Berti, E., Bigongiari, G., Bongi, M., Brogi, P., Bruno, A., Cannady, N., Checchia, C., Cherry, M., Collazuol, G., Ficklin, A., Guzik, T., Ichimura, M., Israel, M., Kasahara, K., Kawakubo, Y., Krizmanic, J., Messineo, A., Mitchell, J., Miyake, S., Mori, M., Mori, N., Motz, H., Munakata, K., Pacini, L., Palma, F., Papini, P., Rauch, B., Ricciarini, S., \& Sakamoto, T. (2021). CALET Observations during the First 5 Years on the ISS. Physics of Atomic Nuclei, 84 (6), 985-994. https://doi.org/10.1134/ S1063778821130214

This Article is brought to you for free and open access by the Department of Physics \& Astronomy at LSU Digital Commons. It has been accepted for inclusion in Faculty Publications by an authorized administrator of LSU Digital Commons. For more information, please contact ir@lsu.edu. 


\section{Authors}

P. S. Marrocchesi, O. Adriani, Y. Akaike, Y. Asaoka, E. Berti, G. Bigongiari, M. Bongi, P. Brogi, A. Bruno, N. Cannady, C. Checchia, M. L. Cherry, G. Collazuol, A. W. Ficklin, T. G. Guzik, M. Ichimura, M. H. Israel, K. Kasahara, Y. Kawakubo, J. F. Krizmanic, A. M. Messineo, J. W. Mitchell, S. Miyake, M. Mori, N. Mori, H. M. Motz, K. Munakata, L. Pacini, F. Palma, P. Papini, B. F. Rauch, S. B. Ricciarini, and T. Sakamoto 
This work was written as part of one of the author's official duties as an Employee of the United States Government and is therefore a work of the United States Government. In accordance with 17 U.S.C. 105 , no copyright protection is available for such works under U.S. Law.

Public Domain Mark 1.0

https://creativecommons.org/publicdomain/mark/1.0/

Access to this work was provided by the University of Maryland, Baltimore County (UMBC)

ScholarWorks@UMBC digital repository on the Maryland Shared Open Access (MD-SOAR) platform.

\section{Please provide feedback}

Please support the ScholarWorks@UMBC repository by emailing scholarworks-group@umbc.edu and telling us what having access to this work means to you and why it's important to you. Thank you. 


\title{
CALET Observations during the First 5 Years on the ISS
}

\author{
P. S. Marrocchesi 1),2)*, O. Adriani ${ }^{3), 4)}$, Y. Akaike ${ }^{5), 6)}$, Y. Asaoka ${ }^{7), 8)}$, E. Berti ${ }^{3), 4),}$ \\ G. Bigongiari ${ }^{1), 2)}$, M. Bongi ${ }^{3), 4)}$, P. Brogi ${ }^{1), 2)}$, A. Bruno ${ }^{9)}$, N. Cannady ${ }^{5), 6)}$, \\ C. Checchia ${ }^{3), 4)}$, M. L. Cherry $^{10)}$, G. Collazuol ${ }^{11), 12)}$, A. W. Ficklin ${ }^{10)}$, T. G. Guzik ${ }^{10)}$, \\ M. Ichimura ${ }^{13)}$, M. H. Israel ${ }^{14)}$, K. Kasahara ${ }^{15)}$, Y. Kawakubo ${ }^{10)}$, J. F. Krizmanic ${ }^{5), 6)}$, \\ A. M. Messineo ${ }^{16), 2)}$, J. W. Mitchell ${ }^{17)}$, S. Miyake ${ }^{18)}$, M. Mori ${ }^{19)}$, N. Mori ${ }^{4)}$, \\ H. M. Motz ${ }^{20)}$, K. Munakata ${ }^{21)}$, L. Pacini'), F. Palma ${ }^{22), 23)}$, P. Papini ${ }^{4)}$, B. F. Rauch ${ }^{14)}$, \\ S. B. Ricciarini ${ }^{24), 4)}$, T. Sakamoto ${ }^{25)}$, M. Sasaki ${ }^{5), 6)}$, F. Stolzi ${ }^{1), 2)}$, A. Sulaj ${ }^{1), 2)}$, \\ T. Tamura ${ }^{26)}$, S. Torii ${ }^{7), 8), 27)}$, J. P. Wefel ${ }^{10)}$, K. Yoshida ${ }^{15)}$, and W. V. Zober ${ }^{14)}$ \\ (for the CALET Collaboration)
}

Received July 28, 2021; revised July 28, 2021; accepted July 28, 2021

\begin{abstract}
The CALorimetric Electron Telescope CALET is collecting science data on the International Space Station since October 2015 with excellent and continuous performance. Energy is measured with a deep homogeneous calorimeter (1.2 nuclear interaction lengths, 27 radiation lengths) preceded by an imaging pre-shower ( 3 radiation lengths, $1 \mathrm{~mm}$ granularity) providing tracking and $10^{-5}$ electron/proton discrimination. Two independent sub-systems identify the charge $Z$ of the incident particle from proton to iron and above $(Z<40)$. CALET measures the cosmic-ray electron + positron flux up to $20 \mathrm{TeV}$, gamma rays up to $10 \mathrm{TeV}$, and nuclei up to the $\mathrm{PeV}$ scale. In this paper, we report the on-orbit performance of the instrument and summarize the main results obtained during the first 5 years of operation, including the electron + positron energy spectrum and the individual spectra of protons, heavier nuclei and iron. Solar modulation and gamma-ray observations are also concisely reported, as well as transient phenomena and the search for gravitational wave counterparts.
\end{abstract}

DOI: $10.1134 / \mathrm{S} 1063778821130214$

${ }^{1)}$ Department of Physical Sciences, Earth and Environment, University of Siena, 53100 Siena, Italy.

${ }^{2)}$ INFN Sezione di Pisa, Polo Fibonacci, 56127 Pisa, Italy.

${ }^{3)}$ Department of Physics, University of Florence, 50019 Sesto Fiorentino, Italy.

${ }^{4)}$ INFN Sezione di Florence, 50019 Sesto Fiorentino, Italy.

${ }^{5)}$ CRESST and Astroparticle Physics Laboratory NASA/GSFC, Greenbelt, MD 20771, USA.

${ }^{6)}$ Department of Physics, University of Maryland, Baltimore County, 1000 Hilltop Circle, Baltimore, MD 21250, USA.

${ }^{7)}$ Research Institute for Science and Engineering, Waseda University, 3-4-1 Okubo, Shinjuku, Tokyo 169-8555, Japan.

${ }^{8)}$ JEM Utilization Center, Human Spaceflight Technology Directorate, Japan Aerospace Exploration Agency, 2-1-1 Sengen, Tsukuba, Ibaraki 305-8505, Japan.

${ }^{9}$ Heliospheric Science Division, NASA/Goddard Space Flight Center - Code 672, Bldg. 21, Room 137D, Greenbelt MD 20771, USA.

${ }^{10)}$ Department of Physics and Astronomy, Louisiana State University, 202 Nicholson Hall, Baton Rouge, LA 70803, USA.
${ }^{11)}$ Department of Physics and Astronomy, University of Padova, 35131 Padova, Italy.

${ }^{12)}$ INFN Sezione di Padova, 35131 Padova, Italy.

${ }^{13)}$ Faculty of Science and Technology, Graduate School of Science and Technology, Hirosaki University, 3, Bunkyo, Hirosaki, Aomori 036-8561, Japan.

${ }^{14)}$ Department of Physics, Washington University, One Brookings Drive, St. Louis, MO 63130-4899, USA.

${ }^{15)}$ Department of Electronic Information Systems, Shibaura Institute of Technology, 307 Fukasaku, Minuma, Saitama 337-8570, Japan.

16) University of Pisa, Polo Fibonacci, 56127 Pisa, Italy.

${ }^{17)}$ Astroparticle Physics Laboratory, NASA/GSFC, Greenbelt, MD 20771, USA.

${ }^{18)}$ Department of Electrical and Electronic Systems Engineering, National Institute of Technology, Ibaraki College, 866 Nakane, Hitachinaka, Ibaraki 312-8508 Japan.

${ }^{19)}$ Department of Physical Sciences, College of Science and Engineering, Ritsumeikan University, Shiga 525-8577, Japan.

${ }^{20)}$ International Center for Science and Engineering Programs, Waseda University, 3-4-1 Okubo, Shinjuku, Tokyo 1698555, Japan. 


\section{INTRODUCTION}

CALET is a space experiment on the ISS designed for long-term observations of charged and neutral cosmic radiation. The instrument is managed by an international collaboration led by the Japanese Space Agency (JAXA) with the participation of the Italian Space Agency (ASI) and NASA. It was launched on August 19, 2015 with the Japanese carrier H-IIB, delivered to the ISS by the HTV5 Transfer Vehicle, and installed on the Japanese Experiment Module Exposure Facility (JEM-EF). The science program of CALET addresses several outstanding questions of high-energy astroparticle physics including the origin of cosmic rays (CR), the possible presence of nearby astrophysical CR sources, the acceleration and propagation of primary and secondary elements in the galaxy, and the nature of dark matter. The design of CALET is optimized for high precision measurements of the electron + positron spectrum allowing for an accurate scan of the energy interval already covered by previous experiments and its extension to the region above $1 \mathrm{TeV}$. Given the high energy resolution of CALET for electrons, a detailed study of the spectral shape might reveal the presence of nearby sources of acceleration as well as possible indirect signatures of dark matter $[1,2]$.

With its capability of identifying cosmic rays with individual element resolution, CALET is also carrying out direct measurements of the spectra and relative abundances of light and heavy cosmic nuclei $[3,4]$, from proton to iron, in the energy range from $\sim 50 \mathrm{GeV}(10 \mathrm{GeV} / \mathrm{n})$ for the lighter (heavier) nuclei to several hundred $\mathrm{TeV}$. The abundances of trans-iron elements up to $Z \sim 40$ are studied with a dedicated program of long term observations [5].

\section{THE CALET INSTRUMENT}

CALET is based on a thick calorimetric instrument (30 radiation lengths), designed to achieve electromagnetic shower containment and a large proton

\footnotetext{
${ }^{21)}$ Faculty of Science, Shinshu University, 3-1-1 Asahi, Matsumoto, Nagano 390-8621, Japan.

${ }^{22)}$ University of Rome "Tor Vergata”, 00133 Rome, Italy.

${ }^{23)}$ INFN Sezione di Rome "Tor Vergata”, 00133 Rome, Italy.

${ }^{24)}$ Institute of Applied Physics (IFAC), National Research Council (CNR), 50019 Sesto Fiorentino, Italy.

${ }^{25)}$ College of Science and Engineering, Department of Physics and Mathematics, Aoyama Gakuin University, 5-10-1 Fuchinobe, Chuo, Sagamihara, Kanagawa 252-5258, Japan.

${ }^{26)}$ Kanagawa University, 3-27-1 Rokkakubashi, Kanagawa, Yokohama, Kanagawa 221-8686, Japan.

${ }^{27)}$ School of Advanced Science and Engineering, Waseda University, 3-4-1 Okubo, Shinjuku, Tokyo 169-8555, Japan.

*E-mail: piersimone.marrocchesi@pi.infn.it
}

rejection capability $\left(>10^{5}\right)$. It is longitudinally segmented into a fine grained imaging calorimeter (IMC) followed by a total absorption calorimeter (TASC). The TASC is a $27 X_{0}$ thick homogeneous calorimeter with 12 alternate $X-Y$ layers of lead-tungstate (PWO) logs. The IMC is a sampling calorimeter segmented longitudinally into 16 layers of scintillating fibers (with $1 \mathrm{~mm}^{2}$ square cross-section) readout individually and interspaced with thin tungsten absorbers. Alternate planes of fibers are arranged along orthogonal directions. It can image the early shower profile in the first $3 X_{0}$ and reconstruct the incident direction of cosmic rays with good angular resolution $\left(0.1^{\circ}\right.$ for electrons and better than $0.5^{\circ}$ for hadrons) [1]. The overall thickness of CALET at normal incidence is $\sim 1.3$ proton interaction lengths $\left(\lambda_{I}\right)$. The charge identification of individual nuclear species is performed by a two-layered hodoscope of plastic scintillators (CHD), positioned at the top of the apparatus, providing a measurement of the charge $\mathrm{Z}$ of the incident particle over a wide dynamic range $(Z=1$ to $\sim 40$ ) with sufficient charge resolution to resolve individual elements [6] and complemented by a redundant charge determination via multiple $d E / d x$ measurements in the IMC. The overall CHD charge resolution (in $Z$ units) increase linearly, as a function of the atomic number, from $<0.1$ for protons to $\sim 0.3$ for iron. For the IMC, although the photostatistics available for a single fiber is about one order of magnitude lower than in the case of a single CHD layer, the charge measurement - thanks to the multiple sampling - achieves an excellent performance as shown in Fig. 2 of [7], where the IMC charge resolution is plotted as a function of the atomic number $Z$. The interaction point (IP) is first reconstructed [8] and only the $d E / d x$ ionization clusters from the layers upstream the IP are used to infer a charge value from the truncated-mean of the valid samples. The geometrical factor of CALET is $\sim 0.1 \mathrm{~m}^{2}$ sr and the total weight is $613 \mathrm{~kg}$. The instrument is described in more detail elsewhere [9]. See also the Supplemental Material (SM) of [11].

\section{FLIGHT OPERATIONS AND CALIBRATIONS}

The commissioning of CALET aboard the ISS was successfully completed at the beginning of October 2015. Since then, the instrument has been taking science data continuously with no major interruptions [2]. The on-orbit operations are controlled via the JAXA Ground Support Equipment (JAXAGSE) in Tsukuba by the Waseda CALET Operations Center (WCOC) at Waseda University, Tokyo.

As of April 30, 2021 a total observation time of more than 2027 days was integrated with a live time 


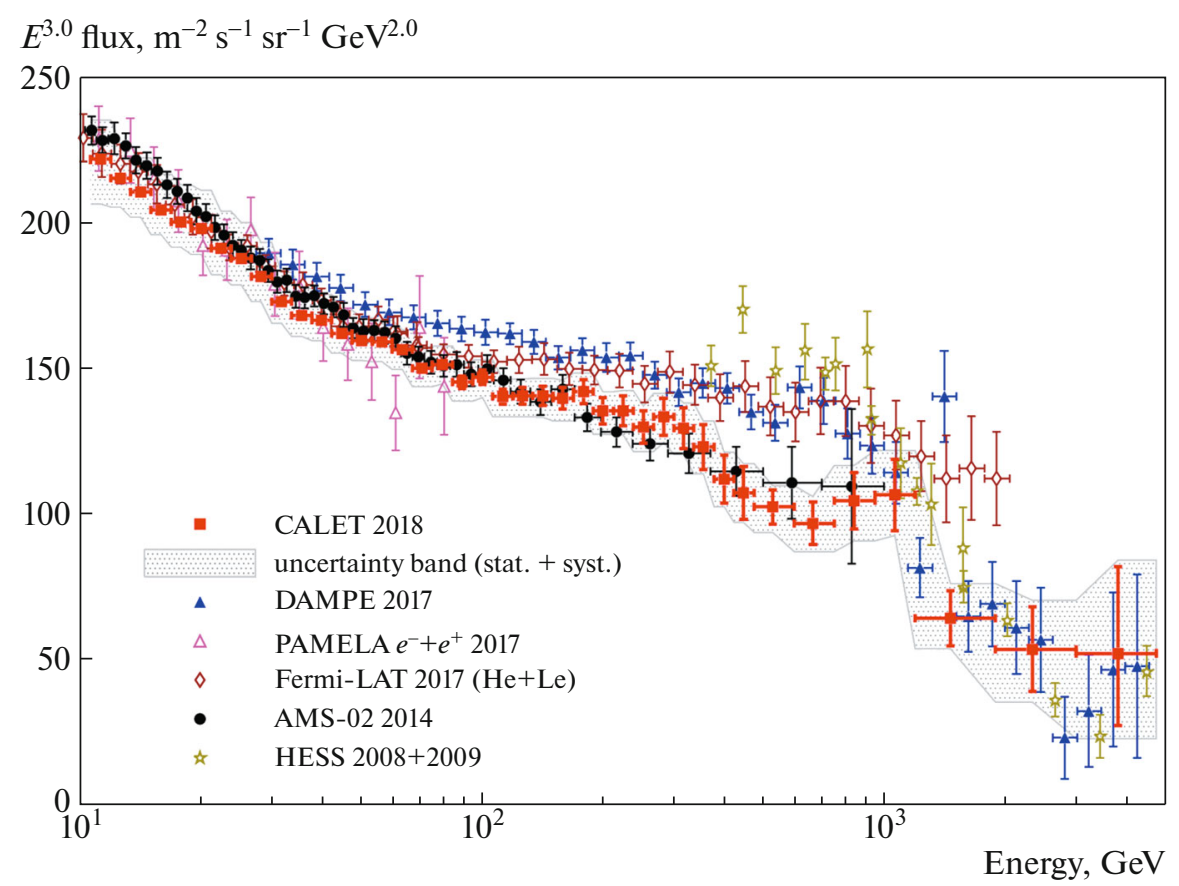

Fig. 1. Direct measurements of the electron + positron flux by space-borne experiments including [12, 13, 14-16] and from ground-based experiments [17, 18]. The CALET 2018 data [13] are shown as red filled circles in the energy interval $11 \mathrm{GeV}$ to $4.8 \mathrm{TeV}$. The width of each bin is shown as a horizontal bar, statistical errors as vertical bars. The gray band indicates the quadratic sum of statistical and systematic errors (not including the uncertainty on the energy scale).

fraction $\sim 85 \%$ of the total time, and $\sim 2.7$ billion events collected above $1 \mathrm{GeV}$. The exposure with the high-energy (HE) trigger mode, designed to maximize the collection power for electrons above $10 \mathrm{GeV}$ and other high-energy shower events, was $\sim 178 \mathrm{~m}^{2}$ sr day.

Energy calibrations of each channel of CHD, IMC, and TASC is performed with penetrating proton and $\mathrm{He}$ particles selected in-flight by a dedicated trigger mode. Raw signals are corrected for light output non-uniformity, gain differences among the channels, position and temperature dependence, as well as temporal gain variations $[9,10]$. Correlations among the four gain ranges for each TASC channel are calibrated with flight data, and responses from consecutive ranges are linked together to provide a seamless transition. In this way, a dynamic range spanning more than six orders of magnitude is achieved, allowing observations from one minimum ionizing particle to $\mathrm{PeV}$ showers.

\section{COSMIC-RAY DIRECT MEASUREMENTS WITH CALET ON THE ISS}

\subsection{The Electron Spectrum}

The CALET collaboration reported their first measurement of the inclusive electron+ positron spectrum in the energy range from $10 \mathrm{GeV}$ to $3 \mathrm{TeV}$ [11] within a fiducial subset of the acceptance. Soon after, the DArk Matter Particle Explorer (DAMPE) collaboration published their all-electron spectrum in the energy interval from $25 \mathrm{GeV}$ to $4.6 \mathrm{TeV}$ [12]. The latter publication was followed by a number of papers speculating about the origin of a possible peak-like structure near 1.4 TeV in DAMPE data. An updated version of the CALET all-electron spectrum was published, covering the energy range from $11 \mathrm{GeV}$ to $4.8 \mathrm{TeV}$ [13] with 780 days of flight data and the full geometrical acceptance. It reported a new analysis with doubled statistics at $E>475 \mathrm{GeV}$ and included one additional energy bin between 3 and $4.8 \mathrm{TeV}$ (Fig. 1). The width of each bin is shown as a horizontal bar, the statistical errors as vertical bars, while the gray band is representative of the quadratic sum of statistic and systematic errors. In the same figure are also plotted direct electron measurements in space including $[12,14-16]$ and from groundbased experiments [17, 18]. A comprehensive study of the systematic uncertainties was performed as described in [11, 13] and Supplemental Material therein. A constant electron identification efficiency of $70 \%$ was achieved above $30 \mathrm{GeV}$, with a proton contamination level of $2-5 \%$ below $1 \mathrm{TeV}$ and $\sim 10-$ $20 \%$ above.

Taking the currently available experimental data at face-value, we notice that the all-electron spectrum 


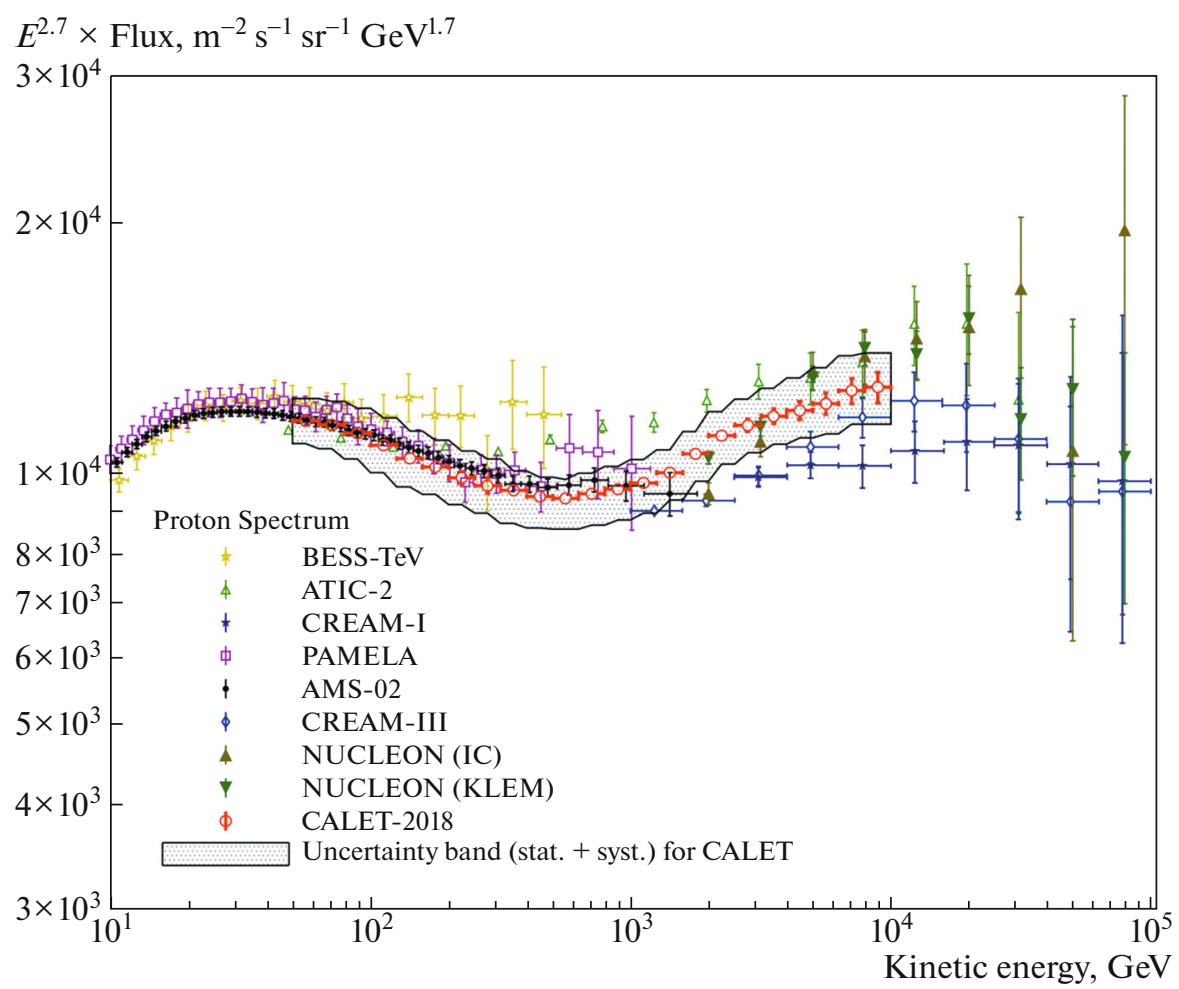

Fig. 2. Cosmic-ray proton spectrum measured by CALET from $50 \mathrm{GeV}$ to $10 \mathrm{TeV}$ published in [25]. The gray band indicates the quadratic sum of statistical and systematic errors.

data seem to fork into two groups of measurements: AMS-02 + CALET and Fermi/LAT + DAMPE, with good consistency within each group, but with only marginal overlap between the two, possibly indicating the presence of unknown systematic errors. CALET spectrum is consistent with AMS-02 below $\sim 1 \mathrm{TeV}$ where both experiments have a good electron identification capability albeit using different detection techniques. CALET observation of a flux suppression above $\sim 1 \mathrm{TeV}$ is consistent with DAMPE within errors. No peak-like structure was found at 1.4 TeV in CALET data, irrespective of the energy binning. After re-binning with the same set of energy bins as DAMPE, an inconsistency between the two measurements emerges with a $4 \sigma$ significance. The latter includes the systematic errors quoted from both experiments.

\subsection{The Proton Spectrum}

Cosmic-ray energies from the $\mathrm{GeV}$ scale to the multi-TeV region have been explored - in separate subranges - by magnetic spectrometers (e.g., BESS-TeV, PAMELA, and AMS-02), calorimeters (e.g., ATIC, CREAM, NUCLEON, and DAMPE) and Cherenkov/Transition Radiation instruments (e.g., TRACER). In the intermediate energy region from 200 to $800 \mathrm{GeV}$ a deviation from a single power-law (SPL) was observed in both proton and helium spectra by CREAM [1921], PAMELA [22, 23 ] and confirmed with high statistics measurements by AMS-02 [24], CALET [25], and DAMPE [26].

The first proton paper published by CALET reported a proton flux measurement where, for the first time, a single space-borne instrument was able to cover the whole interval of proton energies from $50 \mathrm{GeV}$ to $10 \mathrm{TeV}$ thanks to its large dynamic range. The proton flux was extracted from the data collected from October 13, 2015 to August 31, 2018 (1054 days) on the ISS using only $40 \%$ of the total acceptance. A detailed study of the systematic uncertainties was reported in the same paper and in the Supplementary Material therein [25]. This is of particular relevance because CR flux measurements are well known to be affected by relatively large systematic errors, often specific of each instrument. CALET proton data (Fig. 2) are consistent with AMS-02 but extend to higher energies by nearly one order of magnitude, showing a very smooth transition of the spectral index from $-2.81 \pm 0.03$ in the region $50-500 \mathrm{GeV}$ to $-2.56 \pm 0.04(1-10 \mathrm{TeV})$, thereby confirming the existence of a spectral hardening and providing evidence of a deviation from a single power law by more than $3 \sigma$. An update of CALET proton analysis, based on 5 years of data on the ISS, will be presented at the ICRC2021. 


\subsection{The Spectra of Heavier Nuclei}

The observations of a spectral hardening in proton and helium, as well as in carbon and oxygen spectra, [20, 22, 24, 27, 28] have opened a new and unexpected scenario in CR phenomenology. In particular, the high statistics measurements by AMS02, up to a maximum detectable rigidity (MDR) of a few TV, clearly show that primary elements have a very similar rigidity dependence above $\sim 60 \mathrm{GV}$ and that secondary elements (like $\mathrm{Li}, \mathrm{Be}$ and $\mathrm{B}$ ) also show a flux hardening, though with subtle differences that might be attributed to propagation effects (secondaries propagate first as primaries and then as secondaries). Therefore, it is very important to extend the presently available measurements to the multi-TeV region and investigate the energy dependence of the spectral index for individual nuclear species with high accuracy. CALET is carrying out extensive measurements of the energy spectra, relative abundances and secondary-to-primary ratios of cosmic-ray nuclei.

Preliminary CALET results on the $\mathrm{B} / \mathrm{C}$ ratio and on the spectra of heavier nuclei from neon to iron (Fig. 3) were previously reported (see for instance [4, $29,30]$ ). In the following we will focus on the CALET published spectra of $\mathrm{C}, \mathrm{O}$ and $\mathrm{Fe}$.

\subsection{Carbon and Oxygen Spectra}

The energy spectra of carbon and oxygen and their flux ratio were measured by CALET in the energy range from $10 \mathrm{GeV} / n$ to $2.2 \mathrm{TeV} / n$ and published in [31]. CALET observations (Fig. 4) allow to exclude a single power law spectrum for $\mathrm{C}$ and $\mathrm{O}$ at the level of more than $3 \sigma$. A spectral index increase (spectrum flattening) $\Delta \gamma=0.166 \pm 0.042$ (carbon) and $\Delta \gamma=0.158 \pm 0.053$ (oxygen) were measured above $200 \mathrm{GeV} / n$, respectively. The fluxes of $\mathrm{C}$ and $\mathrm{O}$ were found to share the same energy dependence with a constant $\mathrm{C} / \mathrm{O}$ flux ratio $0.911 \pm 0.006$ above $25 \mathrm{GeV} / n$. While the above results are consistent with the ones reported by AMS-02 for the same elements, the absolute normalization of CALET data is significantly lower than AMS-02, but in agreement with previous experiments (including PAMELA for carbon). For more details please refer to [31] and the Supplementary Material therein.

\subsection{The Iron Spectrum}

In a recent paper [32], the CALET collaboration reported their first measurement of the energy spectrum of cosmic-ray iron from $10 \mathrm{GeV} / n$ to $2.0 \mathrm{TeV} / n$. The analysis is based on 4.4 years of observations and the measurement achieves a significantly better precision than most of the existing measurements of the same element. The CALET iron differential spectrum in kinetic energy per nucleon is shown in Fig. 5, where uncertainties including statistical and systematic errors are bounded within a green band. The spectrum is compared with the results from space-based (HEAO3-C2 [33], CRN [34], AMS02 [35], NUCLEON [36]) and balloon-borne experiments (ATIC-02 [37], TRACER [38], CREAM-II [39], Sanriku [40]), as well as ground-based observations (H.E.S.S. [41]). The CALET spectrum is consistent with ATIC-02 and TRACER at low energy and with CRN and HESS at high energy. CALET and NUCLEON iron spectra have similar shapes, while they differ in the absolute normalization of the flux. The latter turns out to be higher for CALET than for CRN by $\sim 10 \%$ on average, while it is lower by $14 \%$ with respect to Sanriku. CALET and AMS02 iron spectra have a very similar shape (Fig. S12 of the Supplemental Material of [32]), but differ in the absolute normalization of the flux by $\sim 20 \%$.

Taking into account the average size of the large systematic errors reported in the literature, CALET data turn out to be consistent with previous measurements within the uncertainty error band, both in spectral shape and normalization. Below $50 \mathrm{GeV} / n$ the spectral shape is found to be similar to the one observed for primaries lighter than iron. Above the same energy, CALET observations are consistent with the hypothesis of an SPL spectrum up to $2 \mathrm{TeV} / n$, i.e., the flattening observed above a few hundred $\mathrm{GeV} /$ nucleon in the $p, \mathrm{C}$, O spectra does not appear to be present in the iron spectrum in the sub-TeV region. Beyond this limit, the uncertainties given by the available statistics and large systematics do not allow yet to draw a significant conclusion on a possible deviation from a single power law. An SPL fit in this region yields a spectral index value $\gamma=-2.60 \pm 0.03$.

\subsection{The Observation of Gamma-Rays}

CALET can identify gamma-rays and measure their energies from $\sim 1 \mathrm{GeV}$ to the $\mathrm{TeV}$ region. Both CHD and the first IMC layers are used in the offline analysis as anti-coincidence against incoming charged particles, taking advantage of the high granularity of the IMC. Gamma-ray candidates are also required to deposit more energy in the bottom IMC layers than in the upper ones where pair conversion takes place. In addition to the HE trigger, CALET implements a LE- $\gamma$ trigger extending the sensitivity to gamma-rays with primary energies down to $\sim 1 \mathrm{GeV}$. This dedicated trigger is activated only at low geomagnetic latitudes (to avoid an increase of the dead-time) and it is also enabled whenever a gammaray burst is triggered by the CGBM [42]. 


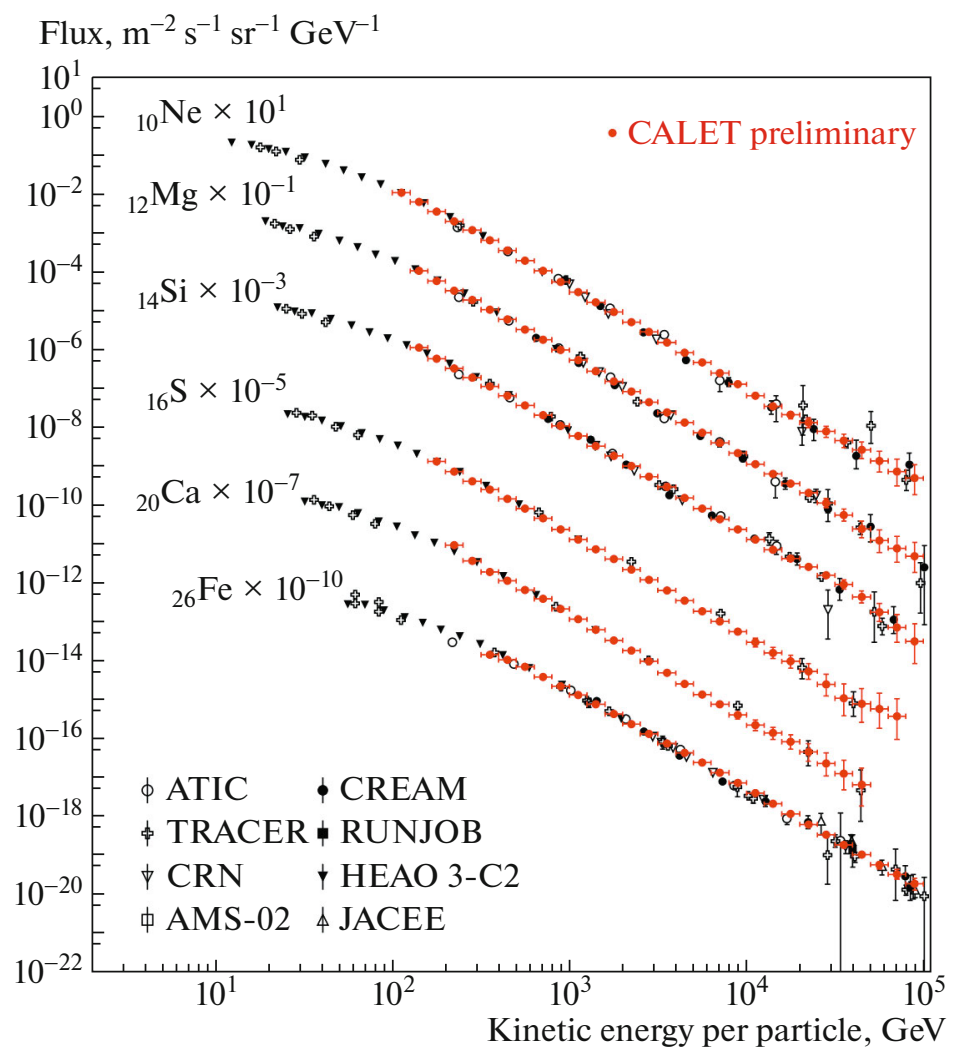

Fig. 3. Preliminary results of energy spectra of heavy primary components of $\mathrm{Ne}, \mathrm{Mg}, \mathrm{Si}, \mathrm{S}$, Ca, and Fe as a function of energy par particle compared with previous observations. The error bars of CALET data [30] represent the statistical uncertainty only.

The first two years of data allowed a complete characterization of the performance of CALET as a gamma-ray instrument, the optimization of the event selection criteria, the determination of the effective area, Point Spread Function (PSF) and absolute pointing accuracy. Measured signals from gammaray bright point sources and diffuse galactic emission were found to be in agreement with simulated results and expectations from Fermi-LAT data [43]. The spectra from sources like Crab, Geminga, and Vela pulsars were measured by CALET and tested for consistency with parameterised LAT spectra. These results confirmed the sensitivity of the calorimeter in observing bright, persistent sources [44]. The gamma-ray sky observed by CALET using the LE$\gamma$ trigger is shown in Fig. 6.

CALET can also detect gamma-ray transients by means of a dedicated Calet Gamma-Ray Burst Monitor (CGBM) operating in the energy range of $7 \mathrm{keV}-20 \mathrm{MeV}$. As of April 2021, $246 \mathrm{GRBs}$ have been detected, $12 \%$ of which were classified as short, with an average rate of $\sim 44.6$ /year.

A search for electromagnetic counterparts of gravitational waves (GW) triggered by LIGO/Virgo was performed with a combined analysis of the CGBM and the calorimeter. Candidate signals compatible with gamma-ray emission were searched for in time intervals of tens of seconds centered on the reported trigger times of GW151226, GW 170104, GW 170608, GW 170814, and GW 170817 events. No signal was detected for all GW events; upper limits on gamma-ray emission were set for GW151226 $(\mathrm{CAL}+\mathrm{CGBM})$ and GW170104 (CAL), while GW 170608, GW170814, GW 170817 turned out to be outside the CALET field-of-view [45, 46].

\section{SUMMARY AND PERSPECTIVES}

CALET was successfully launched on Aug. 19, 2015. The instrument performance has been very stable during all the scientific observation period from Oct. 13, 2015. CALET measurements of the electron spectrum were published in two papers $[11,13]$, the latter with improved statistics and extended energy range from $11 \mathrm{GeV}$ to $4.8 \mathrm{TeV}$. The extension to five years of CALET on-orbit operations provided an increase of the available statistics in the electron observations by a factor $\sim 3$ thereby contributing to a better understanding of the detector and of the systematic errors. A search for possible spectral footprints of 


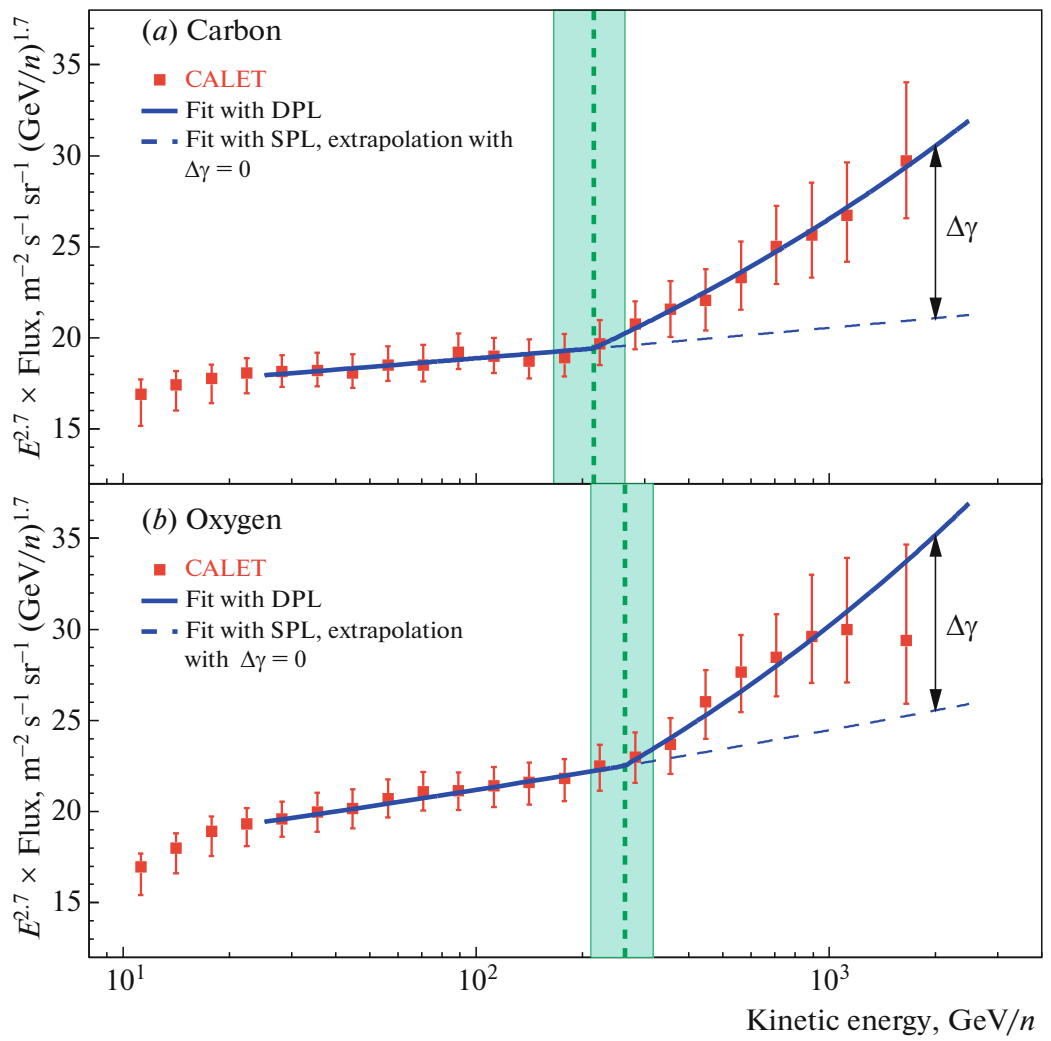

Fig. 4. Fit of the CALET ( $a$ ) C and ( $b$ ) O energy spectra [31] with a Double Power Law (DPL) function (blue line) in the energy range $[25,2000] \mathrm{GeV} / n$. The flux is multiplied by $E^{2.7}$, where $E$ is the kinetic energy per nucleon. Error bars are the sum in quadrature of statistical and systematic uncertainties. The dashed blue lines represent the extrapolation of an Single Power Law function fitted to the data in the energy range $[25,200] \mathrm{GeV} / n . \Delta \gamma$ is the spectral index change above the transition energy $E_{0}$ (vertical green dashed line). The green band shows the uncertainty error on $E_{0}$ from the DPL fit.

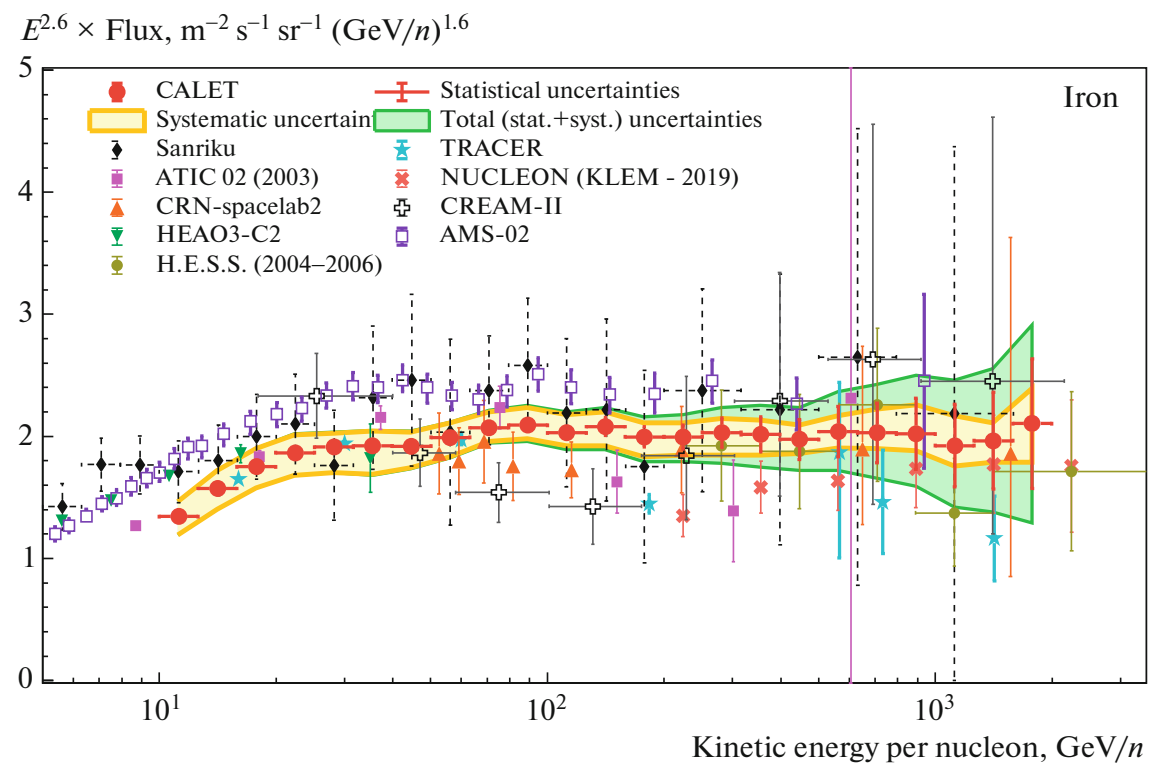

Fig. 5. CALET iron flux [32] as a function of kinetic energy per nucleon in $\mathrm{GeV}$ units (with multiplicative factor $E^{2.6}$ ). The error bars of the CALET data (red filled circles) represent the statistical uncertainty only. The yellow band indicates the quadrature sum of systematic errors, while the green band indicates the quadrature sum of statistical and systematic errors. Also plotted are the data points from other direct measurements [33-41].

nearby electron sources in the region above $\sim 1 \mathrm{TeV}$ is in progress.

The wide dynamic range and excellent charge identification capability allow CALET to measure nuclei in cosmic rays from proton to iron and above, with an energy reach approaching the $\mathrm{PeV}$ scale. 


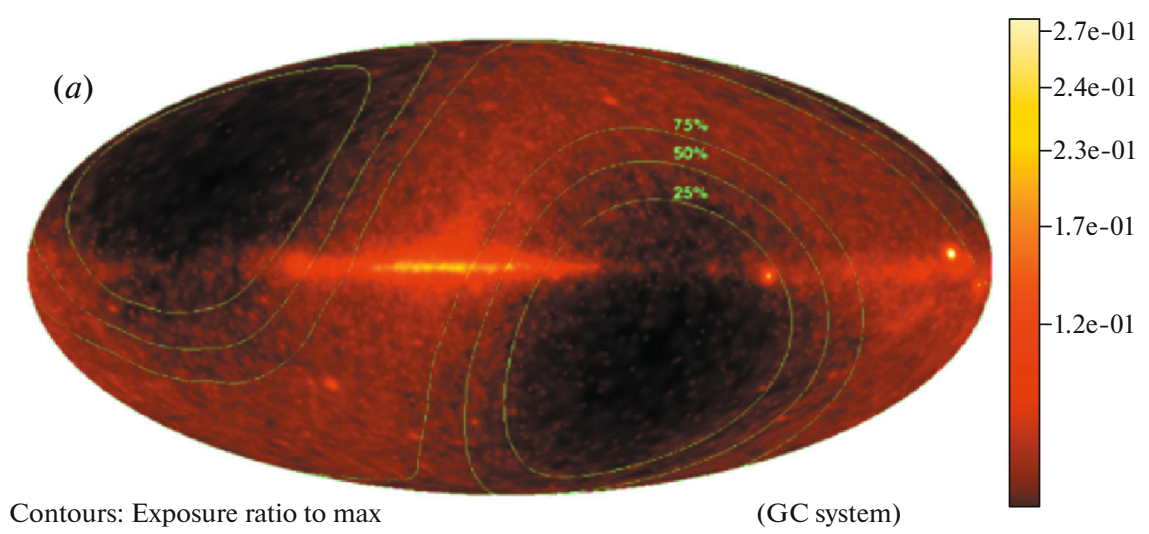

(b)

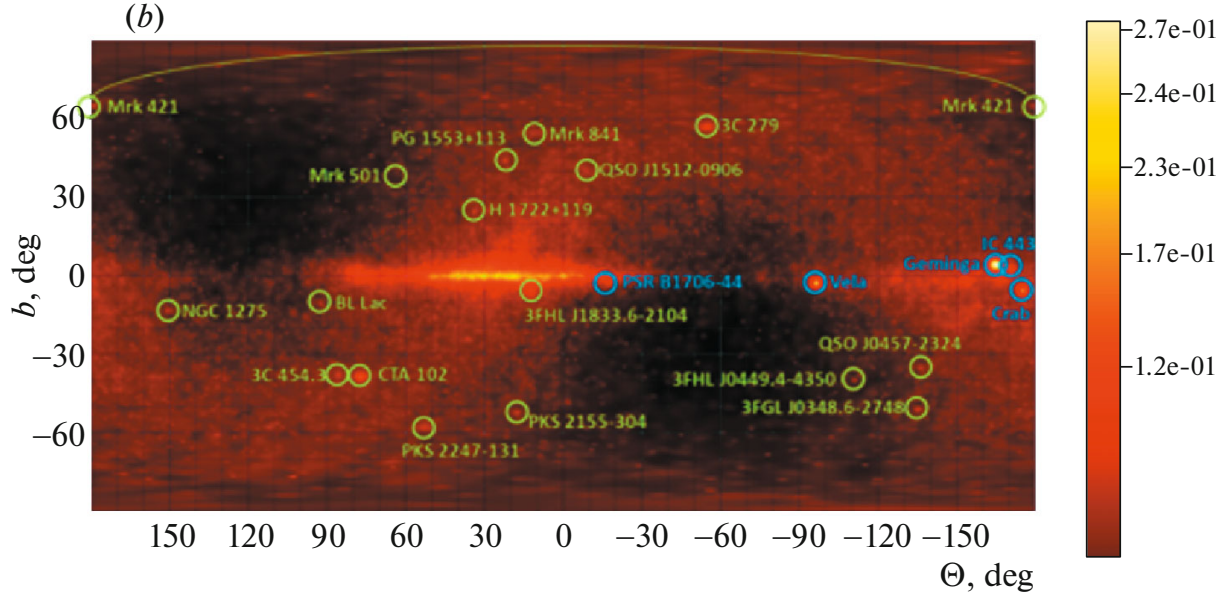

Fig. 6. (a) CALET gamma-ray sky map (observation period: November 1, 2015-July 31, 2020) with the LE- $\gamma$ trigger $(E>1 \mathrm{GeV})$, shown in a Mollweide projection of galactic coordinates. White contours show the relative level of exposure compared to the maximum on the sky; $(b)$ point sources observed during the same period (with $>1 \mathrm{GeV}$ ), including the Crab, Geminga, and Vela pulsars.

The proton spectrum was published up to $10 \mathrm{TeV}$ [25]; $\mathrm{C}$ and $\mathrm{O}$ spectra to $2.2 \mathrm{TeV} / n$ [31], and Fe to $2.0 \mathrm{TeV} / n$ [32]. The spectral index dependence on energy confirmed a spectral hardening for $p, \mathrm{C}$, O with a smooth onset at a few hundred $\mathrm{GeV}$. Measurements of the energy spectra and composition of all primary and secondary nuclei (and of their ratios) are ongoing. The relative abundance of the ultra-heavy nuclei up to $Z=40$ has also been preliminarily analyzed [5].

The performance of the gamma-ray measurements has confirmed CALET's capability to observe the diffuse component and bright point-sources in the gamma-ray sky from $\sim 1 \mathrm{GeV}$ to $100 \mathrm{GeV}$ and above (Fig. 6). The continuous GeV gamma-ray sky observation with CALET complements the coverage by other missions and may help to identify unexplored high-energy emissions from future transient events. The latter phenomena are studied with the CGBM.

Follow-up observations were carried out in the $\mathrm{X}$-ray and gamma-ray band of GW events during LIGO/Virgo observation campaigns [45, 46].
Solar modulation is constantly monitored and studied. Since the start of observations in 2015/10, a steady increase in the $1-10 \mathrm{GeV}$ all-electron flux has been observed to present. In the past two years, the flux has reached the maximum flux observed with PAMELA during the previous solar minimum period [47]. Solar energetic particles (SEP) are also studied at high geomagnetic latitudes.

High statistics detection of $\mathrm{MeV}$ electrons originating from the radiation belt allows the study of relativistic electron precipitation [48]. This is one of the topics of Space Weather studies [49] which were added as additional observational targets for CALET after the start of on-orbit operations.

The so-far excellent performance of the instrument and the outstanding quality of the data suggest that a long-term strategy of CALET observations will most likely contribute to improve our current knowledge of cosmic-ray phenomena. CALET operations on the ISS have been recently approved for an extension to the end of 2024. 


\section{ACKNOWLEDGMENTS}

We gratefully acknowledge JAXA's contributions to the development of CALET and to the operations onboard the ISS. We also wish to express our sincere gratitude to ASI and NASA for their support of the CALET mission.

\section{REFERENCES}

1. S. Torii (for the CALET Collab.), PoS (ICRC2019) $142(2019)$.

2. Y. Asaoka (for the CALET Collab.), PoS (ICRC2019) 001 (2019).

3. S. Torii and P. S. Marrocchesi (for the CALET Collab.), Adv. Space Res. 64, 2531 (2019).

4. Y. Akaike (for the CALET Collab.), PoS (ICRC2019) 034 (2019).

5. B. F. Rauch (for the CALET Collab.), PoS (ICRC2019) 130 (2019).

6. P. S. Marrocchesi, O. Adriani, Y. Akaike, M. G. Bagliesi, A. Basti, G. Bigongiari, S. Bonechi, M. Bongi, M. Y. Kim, T. Lomtadze, P. Maestro, T. Niita, S. Ozawa, Y. Shimizu, and S. Torii, Nucl. Instrum. Methods Phys. Res., Sect. A 659, 477 (2011).

7. P. S. Marrocchesi (for the CALET Collab.), PoS (ICRC2019) 103 (2019).

8. P. Brogi, P. S. Marrocchesi, P. Maestro, and N. Mori (for the CALET Collab.), PoS (ICRC2015) 595 (2015).

9. Y. Asaoka, Y. Akaike, Y. Komiya, R. Miyata, S. Torii, O. Adriani, K. Asano, M. G. Bagliesi, G. Bigongiari, W. R. Binns, S. Bonechi, M. Bongi, P. Brogi, J. H. Buckley, N. Cannady, G. Castellini, et al. (CALET Collab.), Astropart. Phys. 91, 1 (2017).

10. Y. Asaoka, S. Ozawa, S. Torii, O. Adriani, Y. Akaike, K. Asano, M. G. Bagliesi, G. Bigongiari, W. R. Binns, S. Bonechi, M. Bongi, P. Brogi, J. H. Buckley, N. Cannady, G. Castellini, C. Checchia, et al. (CALET Collab.), Astropart. Phys. 100, 29 (2018).

11. O. Adriani, Y. Akaike, K. Asano, Y. Asaoka, M. G. Bagliesi, G. Bigongiari, W. R. Binns, S. Bonechi, M. Bongi, P. Brogi, J. H. Buckley, N. Cannady, G. Castellini, C. Checchia, M. L. Cherry, G. Collazuol, et al. (CALET Collab.), Phys. Rev. Lett. 119, 181101 (2017).

12. G. Ambrosi, Q. An, R. Asfandiyarov, P. Azzarello, P. Bernardini, B. Bertucci, M. S. Cai, J. Chang, D. Y. Chen, H. F. Chen, J. L. Chen, W. Chen, M. Y. Cui, T. S. Cui, A. D'Amone, A. De Benedittis, et al. (DAMPE Collab.), Nature (London, U.K.) 552, $63(2017)$.

13. O. Adriani, Y. Akaike, K. Asano, Y. Asaoka, M. G. Bagliesi, E. Berti, G. Bigongiari, W. R. Binns, S. Bonechi, M. Bongi, P. Brogi, J. H. Buckley, N. Cannady, G. Castellini, C. Checchia, M. L. Cherry, et al. (CALET Collab.), Phys. Rev. Lett. 120, 261102 (2018).
14. O. Adriani, G. C. Barbarino, G. A. Bazilevskaya, R. Bellotti, M. Boezio, E. A. Bogomolov, M. Bongi, V. Bonvicini, S. Bottai, A. Bruno, F. Cafagna, D. Campana, P. Carlson, M. Casolino, G. Castellini, C. De Santis, et al. (PAMELA Collab.), Riv. Nuovo Cim. 40, 473 (2017).

15. S. Abdollahi et al. (The Fermi-LAT Collab.), Phys. Rev. D 95, 082007 (2017).

16. M. Aguilar, D. Aisa, A. Alvino, G. Ambrosi, K. Andeen, L. Arruda, N. Attig, P. Azzarello, A. Bachlechner, F. Barao, A. Barrau, L. Barrin, A. Bartoloni, L. Basara, M. Battarbee, R. Battiston, et al. (AMS Collab.), Phys. Rev. Lett. 113, 221102 (2014).

17. F. Aharonian, A. G. Akhperjanian, U. Barres de Almeida, A. R. Bazer-Bachi, Y. Becherini, B. Behera, W. Benbow, K. Bernlöhr, C. Boisson, A. Bochow, V. Borrel, I. Braun, E. Brion, J. Brucker, P. Brun, R. Bühler, et al. (H.E.S.S. Collab.), Phys. Rev. Lett. 101, 261104 (2008).

18. F. Aharonian, A. G. Akhperjanian, G. Anton, U. Barres de Almeida, A. R. Bazer-Bachi, Y. Becherini, B. Behera, K. Bernlöhr, A. Bochow, C. Boisson, J. Bolmont, V. Borrel, J. Brucker, F. Brun, P. Brun, R. Bühler, et al. (H.E.S.S. Collab.), Astron. Astrophys. 508, 561 (2009).

19. Y. S. Yoon, T. Anderson, A. Barrau, N. B. Conklin, S. Coutu, L. Derome, J. H. Han, J. A. Jeon, K. C. Kim, M. H. Kim, H. Y. Lee, J. Lee, M. H. Lee, S. E. Lee, J. T. Link, A. Menchaca-Rocha, et al., Astrophys. J. 839, 5 (2017).

20. H. S. Ahn, P. Allison, M. G. Bagliesi, J. J. Beatty, G. Bigongiari, J. T. Childers, N. B. Conklin, S. Coutu, M. A. DuVernois, O. Ganel, J. H. Han, J. A. Jeon, K. C. Kim, M. H. Lee, L. Lutz, P. Maestro, et al., Astrophys. J. Lett. 714, L89 (2010).

21. Y. S. Yoon, H. S. Ahn, P. S. Allison, M. G. Bagliesi, J. J. Beatty, G. Bigongiari, P. J. Boyle, J. T. Childers, N. B. Conklin, S. Coutu, M. A. DuVernois, O. Ganel, J. H. Han, J. A. Jeon, K. C. Kim, M. H. Lee, et al., Astrophys. J. 728, 122 (2011).

22. O. Adriani, G. C. Barbarino, G. A. Bazilevskaya, R. Bellotti, M. Boezio, E. A. Bogomolov, L. Bonechi, M. Bongi, V. Bonvicini, S. Borisov, S. Bottai, A. Bruno, F. Cafagna, D. Campana, R. Carbone, P. Carlson, et al. (PAMELA Collab.), Science (Washington, DC, U. S.) 332, 69 (2011).

23. O. Adriani, G. C. Barbarino, G. A. Bazilevskaya, R. Bellotti, M. Boezio, E. A. Bogomolov, M. Bongi, V. Bonvicini, S. Borisov, S. Bottai, A. Bruno, F. Cafagna, D. Campana, R. Carbone, P. Carlson, M. Casolino, et al. (PAMELA Collab.), Astrophys. J. 765, 91 (2013).

24. M. Aguilar, D. Aisa, B. Alpat, A. Alvino, G. Ambrosi, K. Andeen, L. Arruda, N. Attig, P. Azzarello, A. Bachlechner, F. Barao, A. Barrau, L. Barrin, A. Bartoloni, L. Basara, M. Battarbee, et al. (AMS Collab.), Phys. Rev. Lett. 114, 171103 (2015).

25. O. Adriani, Y. Akaike, K. Asano, Y. Asaoka, M. G. Bagliesi, E. Berti, G. Bigongiari, W. R. Binns, S. Bonechi, M. Bongi, P. Brogi, A. Bruno, J. H. Buckley, N. Cannady, G. Castellini, C. Checchia, et al. 
(CALET Collab.), Phys. Rev. Lett. 122, 181102 (2019).

26. Q. An, R. Asfandiyarov, P. Azzarello, P. Bernardini, X. J. Bi, M. S. Cai, J. Chang, D. Y. Chen, H. F. Chen, J. L. Chen, W. Chen, M. Y. Cui, T. S. Cui, H. T. Dai, A. D'Amone, A. De Benedittis, et al. (DAMPE Collab.), Sci. Adv. 5 eaax3793 (2019).

27. M. Aguilar, D. Aisa, B. Alpat, A. Alvino, G. Ambrosi, K. Andeen, L. Arruda, N. Attig, P. Azzarello, A. Bachlechner, F. Barao, A. Barrau, L. Barrin, A. Bartoloni, L. Basara, M. Battarbee, et al. (AMS Collab.), Phys. Rev. Lett. 115, 211101 (2015).

28. M. Aguilar, L. Ali Cavasonza, B. Alpat, G. Ambrosi, L. Arruda, N. Attig, S. Aupetit, P. Azzarello, A. Bachlechner, F. Barao, A. Barrau, L. Barrin, A. Bartoloni, L. Basara, S. Bacsehgmez-du Pree, M. Battarbee, et al. (AMS Collab.), Phys. Rev. Lett. 119, 251101 (2017).

29. P. Maestro, P. S. Marrocchesi, G. Bigongiari, and P. Brogi, Adv. Space Res. 64, 2538 (2019).

30. Y. Akaike (for the CALET Collab.), J. Phys.: Conf. Ser. 1181, 012042 (2019).

31. O. Adriani, Y. Akaike, K. Asano, Y. Asaoka, M. G. Bagliesi, E. Berti, G. Bigongiari, W. R. Binns, M. Bongi, P. Brogi, A. Bruno, J. H. Buckley, N. Cannady, G. Castellini, C. Checchia, M. L. Cherry, et al. (CALET Collab.), Phys. Rev. Lett. 125, 251102 (2020).

32. O. Adriani, Y. Akaike, K. Asano, Y. Asaoka, E. Berti, G. Bigongiari, W. R. Binns, M. Bongi, P. Brogi, A. Bruno, J. H. Buckley, N. Cannady, G. Castellini, C. Checchia, M. L. Cherry, G. Collazuol, et al. (CALET Collab.), Phys. Rev. Lett. 126, 241101 (2021).

33. J. J. Engelmann, P. Ferrando, A. Soutoul, P. Goret, E. Juliusson, L. Koch-Miramond, N. Lund, P. Masse, B. Peters, N. Petrou, and I. L. Rasmussen, Astron. Astrophys. 233, 96 (1990)

34. D. Müller, S. P. Swordy, P. Meyer, J. L'Heureux, and J. M. Grunsfeld, Astrophys. J. 374, 356 (1991).

35. M. Aguilar, L. Ali Cavasonza, M. S. Allen, B. Alpat, G. Ambrosi, L. Arruda, N. Attig, F. Barao, L. Barrin, A. Bartoloni, S. Bacsehgmez-du Pree, R. Battiston, M. Behlmann, B. Beischer, J. Berdugo, B. Bertucci, et al. (AMS Collab.), Phys. Rev. Lett. 126, 041104 (2021)

36. V. Grebenyuk, D. Karmanov, I. Kovalev, I. Kudryashov, A. Kurganov, A. Panov, D. Podorozhny, A. Tkachenko, L. Tkachev, A. Turundaevskiy, O. Vasiliev, and A. Voronin (NUCLEON Collab.), Adv. Space Res. 64, 2546 (2019).

37. A. D. Panov, J. H. Adams, H. S. Ahn, G. L. Bashinzhagyan, J. W. Watts, J. P. Wefel, J. Wu, O. Ganel, T. G. Guzik, V. I. Zatsepin, I. Isbert, K. C. Kim, M. Christl, E. N. Kouznetsov, M. I. Panasyuk,
E. S. Seo, et al. (ATIC Collab.), Bull. Russ. Acad. Sci.: Phys. 73, 564 (2009).

38. M. Ave, P. J. Boyle, F. Gahbauer, C. Hippner, J. R. Horandel, M. Ichimura, D. Müller, and A. Romero-Wolf (TRACER Collab.), Astrophys. J. 678, 262 (2008).

39. H. S. Ahn, P. Allison, M. G. Bagliesi, L. Barbier, J. J. Beatty, G. Bigongiari, T. J. Brandt, J. T. Childers, N. B. Conklin, S. Coutu, M. A. DuVernois, O. Ganel, J. H. Han, J. A. Jeon, K. C. Kim, M. H. Lee, et al. (CREAM Collab.), Astrophys. J. 707, 593 (2009).

40. M. Ichimura, M. Kogawa, S. Kuramata, H. Mito, T. Murabayashi, H. Nanjo, T. Nakamura, K. Ohba, T. Ohuchi, T. Ozawa, Y. Yamada, H. Matsutani, Z. Watanabe, E. Kamioka, K. Kirii, M. Kitazawa, et al., Phys. Rev. D 48, 1949 (1993).

41. F. Aharonian, A. G. Akhperjanian, A. R. Bazer-Bachi, M. Beilicke, W. Benbow, D. Berge, K. Bernlöhr, C. Boisson, O. Bolz, V. Borrel, I. Braun, E. Brion, A. M. Brown, R. Bühler, I. Büsching, S. Carrigan, et al. (H.E.S.S. Collab.), Phys. Rev. D 75, 042004 (2007).

42. K. Yamaoka, A. Yoshida, T. Sakamoto, I. Takahashi, T. Hara, T. Yamamoto, Y. Kawakubo, R. Inoue, S. Terazawa, R. Fujioka, K. Senuma, S. Nakahira, H. Tomida, S. Ueno, S. Torii, M. L. Cherry, S. Ricciarini (CALET Collab.), in Proceedings of the 7 th Huntsville Gamma-Ray Burst Symposium, GRB 2013, eConf C1304143, 41 (2013).

43. N. Cannady, Y. Asaoka, F. Satoh, M. Tanaka, S. Torii, M. L. Cherry, M. Mori, O. Adriani, Y. Akaike, K. Asano, M. G. Bagliesi, E. Berti, G. Bigongiari, W. R. Binns, S. Bonechi, M. Bongi, et al. (CALET Collab.), Astrophys. J. Suppl. Ser. 238, 5 (2018).

44. M. Mori et al. (for the CALET Collab.), PoS (ICRC2019) 586 (2019).

45. O. Adriani, Y. Akaike, K. Asano, Y. Asaoka, M. G. Bagliesi, E. Berti, G. Bigongiari, W. R. Binns, S. Bonechi, M. Bongi, P. Brogi, J. H. Buckley, N. Cannady, G. Castellini, C. Checchia, M. L. Cherry, et al. (CALET Collab.), Astrophys. J. Lett. 863, 160 (2018).

46. O. Adriani, Y. Akaike, K. Asano, Y. Asaoka, M. G. Bagliesi, G. Bigongiari, W. R. Binns, S. Bonechi, M. Bongi, P. Brogi, J. H. Buckley, N. Cannady, G. Castellini, C. Checchia, M. L. Cherry, G. Collazuol, et al. (CALET Collab.), Astrophys. J. Lett. 829, L20 (2016).

47. S. Miyake et al. (for the CALET Collab.), PoS (ICRC2019) 1126 (2019).

48. R. Kataoka, Y. Asaoka, S. Torii, T. Terasawa, S. Ozawa, T. Tamura, Y. Shimizu, Y. Akaike, and M. Mori (CALET Collab.), Geophys. Res. Lett. 43, 4119 (2016).

49. A. Bruno et al. (for the CALET Collab.), PoS (ICRC2019) 1063 (2019). 